\title{
THE ROLE OF SYSTEM TRAINING AND EXPOSURE ON CRASH WARNING EVALUATION
}

\author{
Timothy Brown, Dawn Marshall, Susan Chrysler \\ National Advanced Driving Simulator, University of Iowa \\ Iowa City, Iowa, United States of America \\ timothy-1-brown@uiowa.edu
}

\begin{abstract}
Summary: This research paper explores the role that familiarity with crash warning systems has on the evaluation of those systems. Prior research has not been consistent in it treatment of providing system training and exposure to participants. The potential impact of these differences in methodology on key measures of response and outcome is unknown. Ninety-six participants completed this study that crossed system training with prior exposure to the warning to systematically evaluate these effects for both forward crash warning (FCW) and lane departure warning (LDW) systems evaluations. Prior exposure to the alerts led to changes in engagement with the distraction task for both FCW and LDW events. Training on the system influenced outcomes of the FCW events with less severe outcomes for participants who were aware they had the system. There is also evidence that driver who were aware of the system's presence but did not have prior exposure to it were less likely to complete the experiment successfully. The results of this study point to an advantage in not provide prior system awareness training in terms of longer commitment times to allow the crash warning events to materialize when prior exposure to the alerts is provided.
\end{abstract}

\section{OBJECTIVES}

In 2009, more than 33,000 fatalities occurred in automobile accidents and over 2.2 million others were injured (NHTSA, 2009). With an estimated annual cost of $\$ 230.6$ billion in the year 2000, it has also an immense economic impact on our society (Blincoe et al., 2002). Two of the four most frequent types of crashes are rear-end and lane change crashes. Rear-end crashes account for approximately 28 percent of crashes while lane change crashes stand at 9 percent (NHTSA, 2011). With the mission to save lives, prevent injuries and reduce economic cost incurred by crashes, a significant effort has been set forth by National Highway Traffic Safety Administration (NHTSA) to determine the how crash warning system driver-vehicle interfaces (DVI) should be evaluated and what if any standardization is needed. With a number of crash warning systems from several automobile manufacturers expanding into the marketplace, a better understanding of the trade-offs in system evaluations is needed. In the evaluation of crash warning systems, there have been varieties of approaches used to provide information to the driver about what crash warning system, is present during the evaluation. This paper examines how information provided to the driver influences the evaluation of the system. We divide system familiarity into two components: system training (awareness of the presence of the system) and prior exposure to the system warning. With regard to system training, some evaluations have been conducted without any awareness that a system was present in the vehicle (e.g. Forkenbrock et al., 2011; Lee et al., 2002), while other research have provided training and documentation consistent with what would be an owner's manual on the system (e.g. Lerner et al. 2011; Hollopeter et al, 2012). No training would represent a worst-case scenario, whereas 
training would provide the best case scenario for driver awareness. Additionally, exposure to the system has been variable with some research providing an opportunity to experience the system (e.g. Lerner et al, 2011; Hollopeter et al, 2012) and others providing no context to the warning (e.g. Forkenbrock et al., 2011, Lee et al., 2002). To date, no study has methodologically evaluated the impact of these differences in approach. The aim of the study was to examine how differences in familiarity with the FCW and LDW systems effect the ability to evaluate a system DVI. This was accomplished through different levels of training on the system and associated alert modality and whether participants were exposed to the alert in the vehicle prior to receiving it during an FCW or LDW event.

\section{METHODS}

The experimental design for this study was a mixed between/within-subject design with two between-subject levels of training, two between-subject levels of exposure, three betweensubject levels of alert modality and two within-subjects levels of event. Two levels of training of the systems were provided to participants during the briefing. The first level was no awareness of a system being present (Not Aware) and the second was training on material similar to that included in a vehicle owner's manual (Aware). The two levels of exposure were no exposure prior to the imminent event and exposure at the beginning of the drive. The choreographed exposure was implemented during the first few minutes of the drive by instructing participants to approach a lead vehicle until they experienced the FCW alert and asking the participants to drive toward the lane lines where they experienced the LDW alert. Although there are many different alert DVIs that could be evaluated, three levels of alert modality were chosen haptic, auditory, and haptic/active to provide a cross-section of the approaches and to compare with existing data. For the FCW system, these were seat belt tensioner, repeated beeps, and brake pulse. For the LDW system, these were steering wheel vibration, acoustic alert, and steering wheel torque. The two levels of event were first presentation and second presentation for the FCW and LDW events. Periodically, a secondary number recall task was presented to the driver to take attention from the forward roadway that would allow LDW and FCW events to be triggered. The number recall task was selected based on a comparison between several tasks on effectiveness of shifting the driver's attention from the road for crash events (Nadler et al, 2013). The SAS General Linear Models (GLM) procedure was used to perform an Analysis of Variance (ANOVA) on the parametric dependent measures.

\section{Dependent Measures}

The following measures were analyzed for the FCW and LDW events: time to end of visual commitment from warning, duration of first visual commitment, minimum time to collision, brake reaction time from warning, maximum lane exceedance, area of lane exceedance, and steering reaction time from warning. Visual commitment is measured relative to looking away from the forward roadway and toward the number recall task. The start of visual commitment is defined as the first instant the participant's vision departs forward. The end of visual commitment is defined as first instant that the participant's vision returns forward. Visual commitments were determined by video coding from two independent coders. 


\section{Participants}

Ninety-six out of one-hundred and thirty-eight participants between the ages of 35 and 55 with a license for at least two years who drive at least 10,000 miles per year, who are willing to engage in distracting tasks while driving and who do not drive a vehicle with advanced safety systems enrolled and completed all study procedures. These requirements were selected to minimize between-subject variability in the data. Of the forty-two who did not complete the study, twelve withdrew from the study; and thirty were dropped from the study with nine for visual commitments to the number recall task that were too short, seven who did not receive a FCW event, one for being unwilling to engage with the number recall task, thirteen for non-study issues. Participants were compensated $\$ 45$ for completing all study procedures.

\section{Apparatus}

The NADS-1, Figure 1, is located at The UI's Oakdale Campus. It consists of a 24-foot dome in which an entire car is mounted. All participants drove the same 1996 Malibu sedan. The motion system, on which the dome is mounted, provides 400 square meters of horizontal and longitudinal travel and \pm 330 degrees of rotation. Each of the three front projectors has a resolution of $1600 \times 1200$; the five rear projectors have a resolution of $1024 \times 768$. The edge blending between projectors is five degrees horizontal. The NADS produces a thorough record of vehicle state and driver inputs, sampled at $240 \mathrm{~Hz}$.

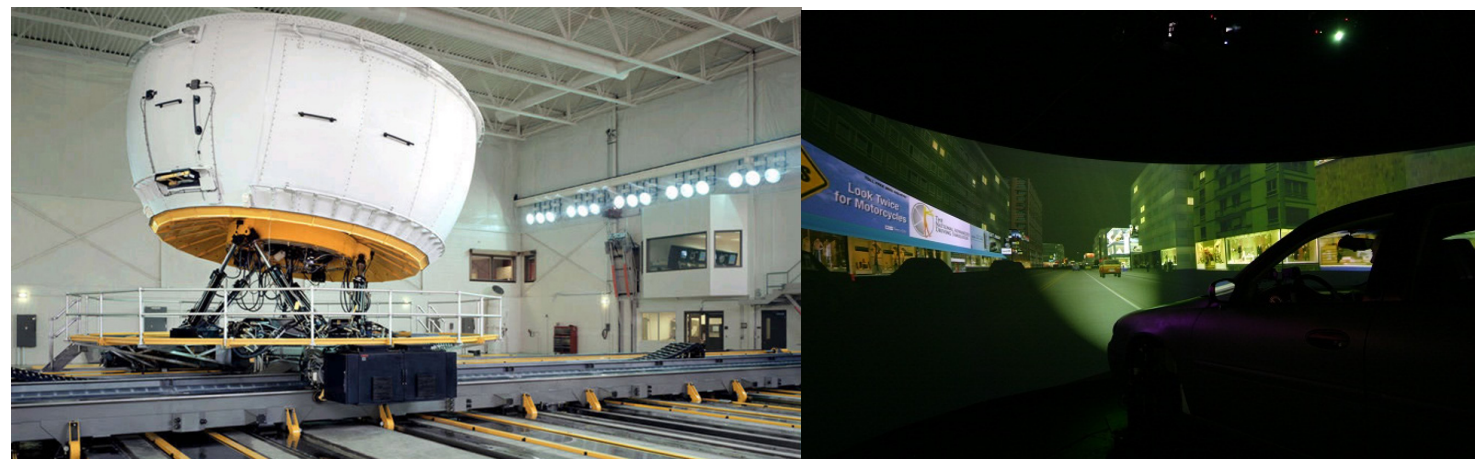

Figure 1. NADS-1 Driving Simulator (left) with a Driving Scene from Inside the Dome (right)

Two displays were mounted in the simulator cab for this study: a monitor to display the numbers task located to the participant's right, and the headway display located near the participant's line of vision. This number recall task display was located to the right of the participant's forward facing position mounted on the rear of the passenger seat headrest and adjusted for each participant so that the display was located at an angle of at least 90 degrees (see Figure 2). One second after receiving the instruction to begin, a series of five random single-digit numbers was presented for $472 \mathrm{~ms}$ each. The participant was to repeat them aloud in the correct order to the experimenter following the task (Forkenbrock et al., 2011). The headway display was mounted on the dashboard centered to the right of the forward line of sight of the participant (see Figure 3). The display provided an analog representation of the accuracy of the headway maintenance. 


\section{Experimental Procedures}

Participants were recruited for this study using the NADS subject registry and through emails to the university community. Individuals who expressed interest were screened over the phone to provide a study overview and to determine eligibility. Upon arrival, the elements of participation were described and participants provided informed consent. Participants were told that the goal of the research was to evaluate several new in-vehicle technologies, when in fact their response to surprise LDW and FCW events was being evaluated. Two different training presentations were used for this study based upon the experimental conditions. Each provided detail on the distraction tasks to be performed. They differed in the description of systems present in the car based on the experimental conditions for each participant. Additionally, participants practiced the number recall task before going into the simulator to become comfortable with performing the task. Participants were prompted to engage with the task consistent with the method used in the simulator. They were asked to practice until they could perform the task successfully by recalling all five numbers correctly with no sequence errors

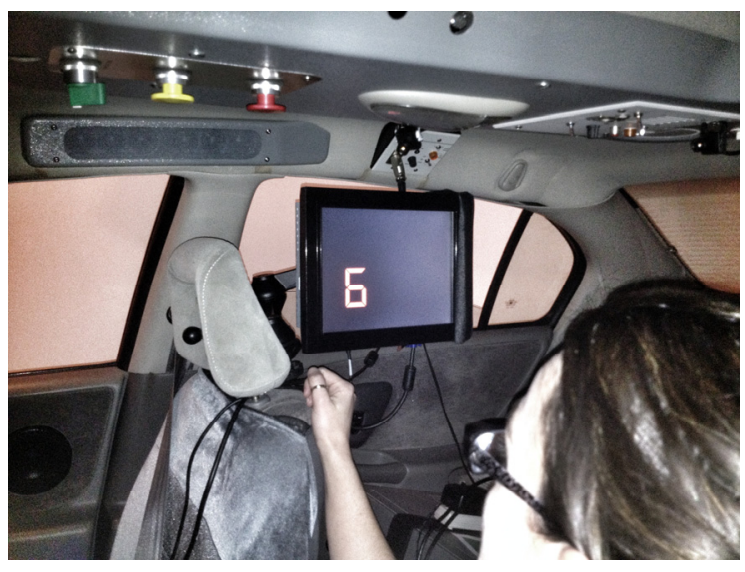

Figure 2. Number Recall

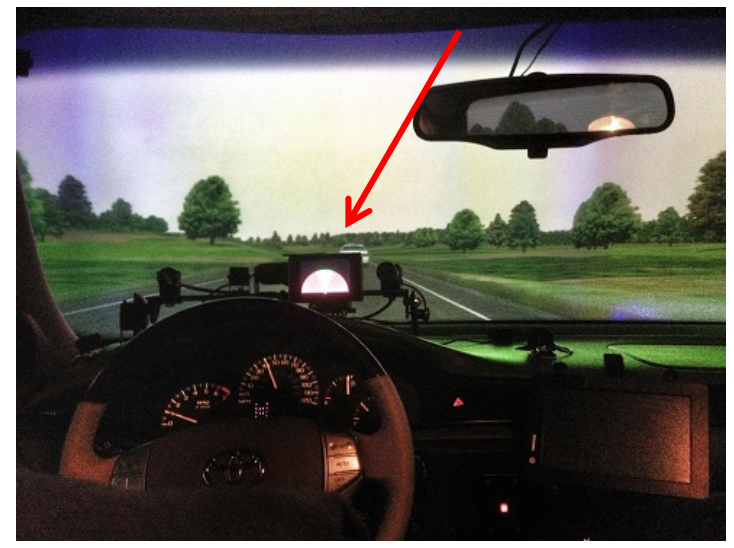

Figure 3. Revised Headway Display Location

\section{Scenarios}

The driving database started with a practice portion in a low-speed urban environment followed by a two-lane rural roadway with intersections where the speed limit alternated between 40 and $55 \mathrm{mph}$. Throughout the drive, they were asked to maintain a headway with the lead vehicle such that the headway remained in the green zone of the display. The drive included 15 occurrences of the number recall task without a related LDW or FCW event, three occurrences where the task was coupled with a potential LDW event and two occurrences where the task was coupled with an FCW event. The drive was designed so that the LDW events occurred prior to the FCW events. For both the LDW and FCW events, the distraction task began 2.08 seconds after the instruction were started to the driver. For the FCW events, a stopped lead vehicle was revealed by the lead vehicle changing lanes to pass such that the warning sounded 3.4 seconds after the start of the instructions and with a TTC of 2.1 seconds. For the LDW event, once the task began, a lateral push was provided toward the closest lane boundary and an alert was provided at 4 
inches from the lane boundary. The push was implemented such that there was no motion feedback associated with the lateral push.

\section{RESULTS}

For response to the alert for lane departure events, there were no effects associated with training about the system or prior exposure to the system. Time to the end of visual commitment was not significantly different for either training or for exposure $(p>0.05)$. There were no interactive effects for training with warning type for time to end of visual commitment. Steering reaction time to the alert was not significantly different for either training or for exposure $(\mathrm{p}>0.05)$. Additionally there were no interactions for either training or exposure with warning type for steering reaction time. Additionally, there were no significant differences based on training about the system for any of the measures of visual commitment or response. However, there were significant differences for exposure. Significant differences were observed for visual commitment duration $(\mathrm{p}=0.0077)$, see, where commitment durations were longer by $\sim 250 \mathrm{~ms}$ with prior exposure to the alert.

When looking at the endpoints for response to the alert for forward crash events, there were two significant effects associated with training about the system and none associated with prior exposure to the system. Time to the end of visual commitment was not significantly different for either training or for exposure $(\mathrm{p}>0.05)$; however there was a significant two-way interaction between exposure, and event $(\mathrm{p}=0.0366)$. There were no interactive effects for training with warning type for time to end of visual commitment. As can be seen in Figure 5, with prior exposure, subjects maintained visual commitment longer for the first forward crash event than for the second event. Brake reaction time to the alert was significantly different by training condition $(p=0.0082)$ but not for exposure $(p>0.05)$. As can be seen in Figure 6 , subjects who had been trained and were aware of the system responded $\sim 171 \mathrm{~ms}$ faster than subjects who were not aware of the system. There were no interactive effects for training or exposure with warning type for brake reaction time. When looking at the other measures that might be influenced by training and exposure, there was a significant difference relative to training for minimum timeto-collision, but no significant differences for exposure. For minimum TTC, subjects with prior exposure to the alert had significantly greater minimum TTCs $(p=0.0238)$. Another consideration in conducing these evaluations is the efficiency of the protocol. The amount of data needing replaced is a good surrogate measure of this. The number of subject needing replacement due to lost data by condition were seven for no exposure-no training, six for exposure-no training, 13 for no exposure-training, and 2 for exposure-training. These numbers represent how many additional participants needed to run through the protocol in each of those conditions to reach the required sample size of 24 participants per cell.

\section{CONCLUSIONS}

When considering how much familiarity subjects should have with the system in order to best assess the effectiveness of the warning system DVI, there are the practical consideration of what occurs in the real world and how different types of familiarity affect the ability to differentiate between DVIs of different effectiveness. The first consideration is largely philosophical as different drivers will have different familiarities with the systems in their vehicle, and cannot be 
assessed by the data. Providing subjects with awareness that the crash warning systems were present in the car through a pre-drive training presentation resulted in no differences in engagement with the secondary task or driver response for the lane departure events. When considering the forward crash events, the presence of the pre-drive training resulted in greater minimum TTCs and faster brakes applications. For neither type of event was there an interaction with type of DVI used for the warning system. Providing subjects with prior exposure to the warning system during the drive resulted in differences in visual commitment for the lane departure events. These differences were that with prior exposure, subjects had longer engagement times with the secondary task, but that there was no resultant difference in end of visual commitment in response to the alert. When considering the forward crash events, there were no differences based solely on exposure, but when considering whether it was the first or second event, subjects with prior exposure waited longer to end their visual commitment. When considering data needing replacement, providing exposure to the alert appears to reduce the amount of data needing replacement, but there is a mixed effect of providing prior training about the system.

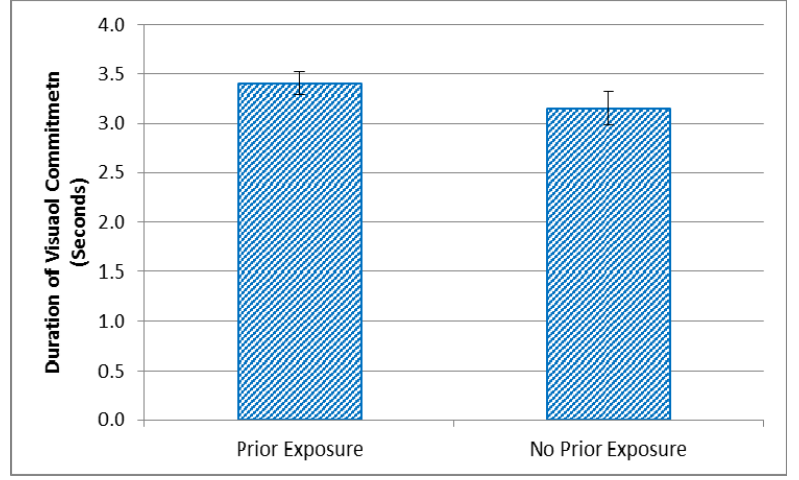

Figure 4. Visual Commitment Times for Lane Departure Events

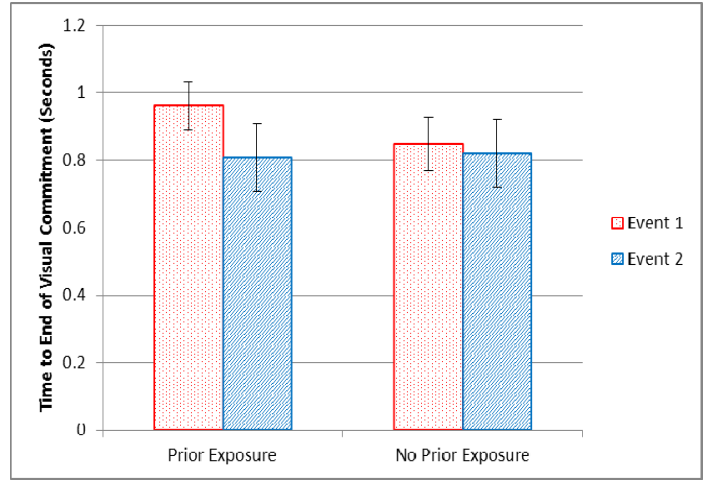

Figure 5. Effect of Prior Exposure and Event on End of Visual Commitment

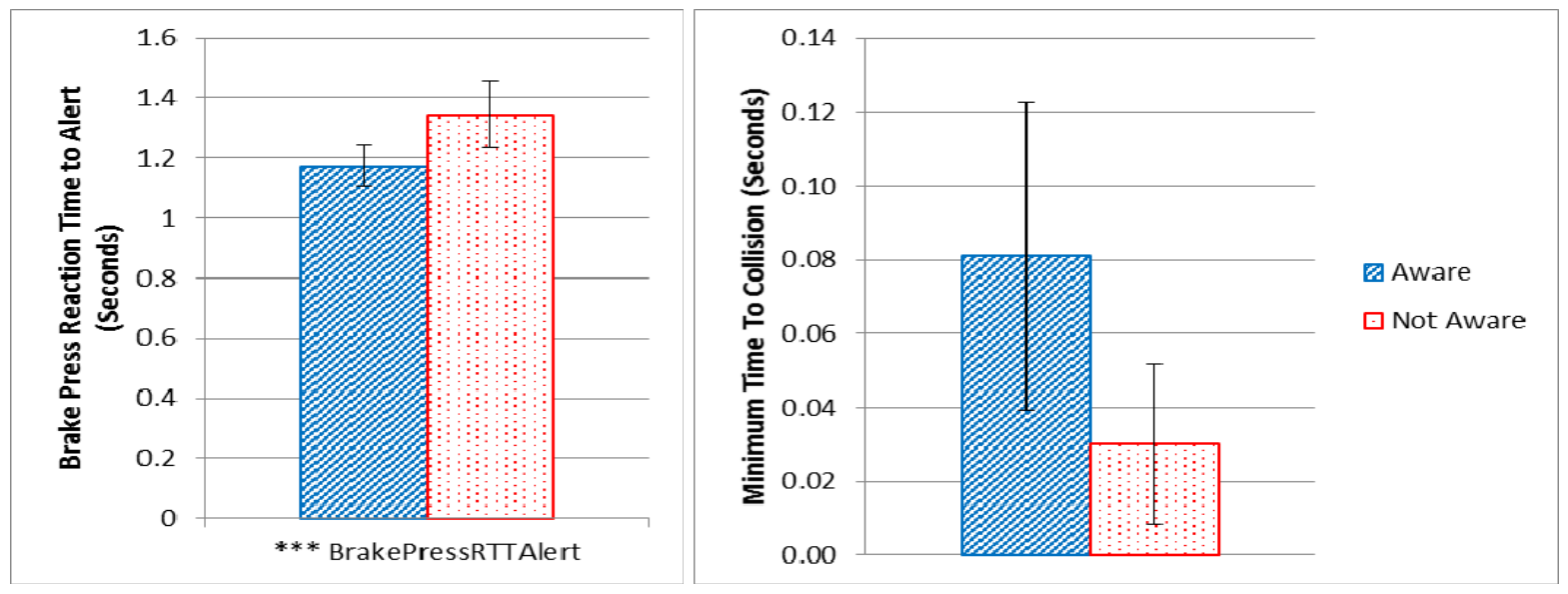

Figure 6. Main effect of System Training on Brake Reaction Time and Minimum TTC

Based on the findings, an argument could be made for either providing the subject with this information or not prior to the study drive. Knowing about the warning in detail seems to allow 
drivers to respond more quickly. No overall guidance can be provided for whether to provide training based on this data. When looking at trends in the data for engagement, the strongest argument would be to not provide training based on the trend toward towards shorter engagements with the secondary task by approximately $120 \mathrm{~ms}$. Although this difference is not significant, caution would dictate that absent a dis-benefit, the safer course would be to not provide prior system awareness training to evaluate warning DVIs. The results of this study point to an advantage in terms of longer commitment times to allow the crash warning events to materialize when prior exposure to the alerts is given at the beginning of the drive.

\section{LIMITATIONS}

Limitations of the study include that the age range is restricted to a single age range, which helps minimize variability but may limit the generalizability to other ages; and that focuses on a short term evaluation of warning system interfaces does not provide guidance into how responses may change based on a longer term exposure.

\section{ACKNOWLEDGMENTS}

The National Highway Traffic Safety Administration funded this research under contract number: DTNH22-11-D-00237, Task Order 2. These findings represent the views of the authors. The authors would like for further recognize Eric Traube, NHTSA, and Neil Lerner, Westat, for their contribution to this research.

\section{REFERENCES}

Blincoe et al. (2002) The Economic Impact of Motor Vehicles Crashes. Washington, DC: National Highway Traffic Safety Administration.

Forkenbrock et al. (2011) A Test Track Protocol for Assessing Forward Collision Warning Driver-Vehicle Interface Effectiveness. (DOT HS 811 501) Washington, DC: National Highway Traffic Safety Administration.

Hollopeter, N., Brown, T. \& Thomas, G. (2012). Differences in Novice and Experienced Driver Response to Lane Departure Warnings that Provide Active Intervention. Proceedings of the Human Factors and Ergonomics Society $56^{\text {th }}$ Annual Meeting. Boston.

Lee, J. D., McGehee D. V., Brown T. L., \& Reyes, M. L. (2002).Collision warning timing, driver distraction, and driver response to imminent rear-end collisions in a high-fidelity driving simulator. Human Factors, 44, 314-334.

Lerner et al. (2011). Crash Warning Interface Metrics: Final Report. (DOT HS 811 470a) Washington, DC: National Highway Traffic Safety Administration.

Nadler, E., Traube, E., Lerner, N., Jenness, J., Brown, T., Baldwin, C., Chiang, D., \& Forkenbrock, G. (2013). Development of Crash Warning Interface Metrics (CWIM). 23rd International Technical Conference on the Enhanced Safety of Vehicles. Seoul, South Korea

NHTSA (2009). Traffic Safety Facts. (DOT HS 811 173) Washington, DC: National Highway Traffic Safety Administration. 